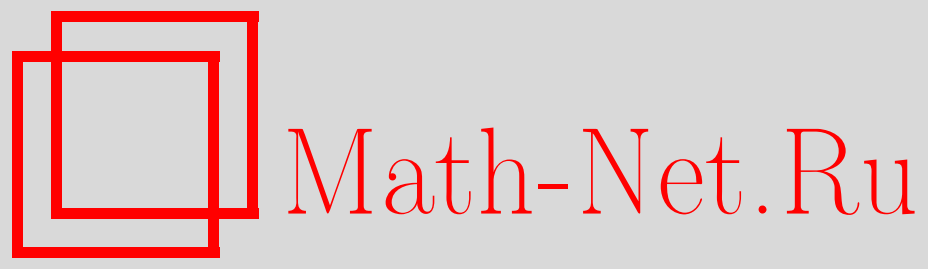

Н. А. Ерзакова, О разрешимости уравнений с частично аддитивными операторами, Функи. анализ и его прил., 2010, том 44, выпуск 3, 69-72

DOI: https://doi.org/10.4213/faa3004

Использование Общероссийского математического портала MathNet.Ru подразумевает, что вы прочитали и согласны с пользовательским соглашением

http://www . mathnet.ru/rus/agreement

Параметры загрузки:

IP : 54.92 .164 .108

26 апреля 2023 г., 14:15:47

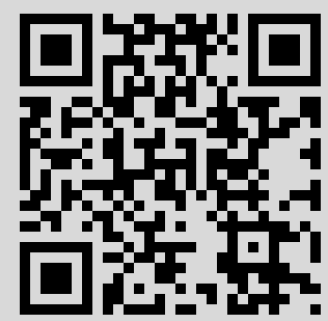


Определим последовательность норм $\left\{\|\cdot\|_{m}\right\}_{m=0}^{\infty}$ на пространстве финитных векторов $c_{00}$. Если $x=\sum_{n} \alpha_{n} e_{n} \in c_{00}$, то

$$
\|x\|_{0}=\max _{n}\left|\alpha_{n}\right|, \quad\|x\|_{m+1}=\max \left(\|x\|_{m}, \frac{1}{2} \max _{c \in \mathscr{A}} \sum_{j=1}^{\operatorname{card} c}\left\|x \chi_{c[j]}\right\|_{m}\right), \quad m \geqslant 0,
$$

где $\chi_{E}-$ характеристическая функция множества $E$. Тогда $c_{00}$ с нормой $\|\cdot\|_{m}$ является нормированной решеткой, инвариантной относительно сдвига, и $\|x\|_{c_{0}} \leqslant\|x\|_{m} \leqslant\|x\|_{l_{1}}\left(x \in c_{00}\right)$. Поскольку $\|x\|_{m+1} \geqslant\|x\|_{m}$ для любого $x$, существует предел $\|x\|_{\mathscr{A}}=\lim _{m \rightarrow \infty}\|x\|_{m}$. Пополнение пространства $c_{00}$ по норме $\|\cdot\|_{\mathscr{A}}$ обозначим через $F$. Нетрудно показать, что для любого $x \in F$

$$
\|x\|_{\mathscr{A}}=\max \left(\|x\|_{c_{0}}, \frac{1}{2} \sup _{c \in \mathscr{A}} \sum_{j=1}^{\operatorname{card} c}\left\|x \chi_{c[j]}\right\|_{\mathscr{A}}\right) .
$$

Теорема 2. Банахова решетка $F$ сепарабельна, инвариантна относительно сдвига, обладает свойствами (RSP), (LSP) и не совпадает с $l_{p}(1 \leqslant p<\infty)$ $u c_{0}$.

Следствие 1. Банахова пара $\left(F, F\left(2^{-k}\right)\right) \quad \mathscr{K}_{u}$-монотонна, но не является равномерным частичным ретрактом никакой весовой $L_{p}$-nарь.

\title{
ЛИТЕРАТУРА
}

[1] Yu. A. Brudnyi, N. Ya. Krugljak, Interpolation Functors and Interpolation Spaces, 1, North-Holland, Amsterdam, 1991. [2] N. J. Kalton, Studia Math., 106:3 (1993), 233277. [3] M. Cwickel, P. Nilsson, in: Interpolation Spaces and Allied Topics in Analysis (Lund, 1984), Lecture Notes in Math., vol. 1070, Springer-Verlag, Berlin, 1984, 5465. [4] А. А. Седаев, Докл. АН СССР, 209:4 (1973), 798-800. [5] В. И. Дмитриев, С. Г. Крейн, В. И. Овчинников, в кн.: Геометрия линейных пространств и теория операторов, Ярослав. гос. ун-т, Ярославль, 1977, 31-74. [6] Й. Берг, Й. Лёфстрём, Интерполячионные пространства. Введение, Мир, М., 1980. [7] P. G. Casazza, T. J. Shura, Tsirelson's Space, Lecture Notes in Math., vol. 1363, Springer-Verlag, Berlin, 1989. [8] T. Figiel, W. B. Johnson, Compositio Math., 29 (1974), 179-190.

Самарский госуниверситет

e-mail: astashkn@ssu.samara.ru

Самарский госуниверситет

e-mail: ktikhomirov@yandex.ru
Поступило в редакцию 18 сентября 2008 г.

\section{О разрешимости уравнений \\ с частично аддитивными операторами}

\author{
(c) 2010. H. A. EpзаковA
}

1. Введение. Пусть $\Omega \subset \mathbb{R}^{n}$, причем $\mu(\Omega)<\infty, \mu$ - непрерывная мера; $u_{0}, u_{01}$ - измеримые функции с положительными значениями (единицы), $\theta-$ функция, равная нулю п. в., $P_{D} u$ - функция, совпадающая с $u$ для всех $s \in D$ 
и равная 0 , если $s \notin D$, для произвольной измеримой функции $u$ на $\Omega$ и произвольного измеримого подмножества $D \subset \Omega ; D\left(u, T, u_{0}\right)=\left\{s:|u(s)|>T u_{0}\right\}$ для произвольного числа $T>0 ; M\left(u_{0}\right)$ - банахово пространство всех измеримых комплекснозначных функций на $\Omega$, для которых имеет смысл и конечна норма $\|u\|_{M\left(u_{0}\right)}=\inf \left\{\lambda:|u| \leqslant \lambda u_{0}\right.$ п. в. $\}[1] ; E, E_{1}$ - правильные пространства функций на $\Omega$, т. е. идеальные пространства с абсолютно непрерывной нормой (см., например, [1]). Примерами правильных пространств, наряду с пространствами Лоренца и пространствами Орлича, $N$-функция которых удовлетворяет $\Delta_{2}$-условию, могут служить пространства $L_{p}\left(\Omega, u_{0}\right)(1 \leqslant p<\infty)$ с нормой $\|u\|_{L_{p}\left(\Omega, u_{0}\right)}=\left\|u / u_{0}\right\|_{L_{p}(\Omega)}$. Напомним также, что оператор $A: E \rightarrow E_{1}$ называется частично аддитивным, если $A\left(v_{1}+\cdots+v_{k}\right)=A\left(v_{1}\right)+\cdots+A\left(v_{k}\right)-(k-1) A(\theta)$ для всех функций $v_{1}, \ldots, v_{k}$ из $E$, которые имеют непересекающиеся носители. Примерами частично аддитивных операторов могут служить, помимо линейных операторов, нелинейный оператор суперпозиции и интегральный оператор Урысона [2]. Для произвольного подмножества $U \subset E$, неограниченного по норме пространства $M\left(u_{0}\right)$, положим

$$
\varkappa(U, A)=\varlimsup_{T \rightarrow \infty} \sup _{u \in U,\left\|P_{D\left(u, T, u_{0}\right)} u\right\|_{E} \neq 0} \frac{\left\|A\left(P_{D\left(u, T, u_{0}\right)} u\right)\right\|_{E_{1}}}{\left\|P_{D\left(u, T, u_{0}\right)} u\right\|_{E}} .
$$

Если $U \subset E-$ ограниченное по норме пространства $M\left(u_{0}\right)$ подмножество в $E$, то полагаем $\varkappa(U, A)=0$. Напомним, что мерой некомпактности Хаусдорфа $\chi_{E}(U)$ множества $U$ называется нижняя грань всех $\varepsilon>0$, при которых $U$ имеет в $E$ конечную $\varepsilon$-сеть. Непрерывный оператор $A: E \rightarrow E_{1}$ называется уплотняющим, если для любого ограниченного подмножества $U$ из $E$, замыкание которого некомпактно, выполняется неравенство $\chi_{E_{1}}(A(U))<\chi_{E}(U)$. Непрерывный оператор $A: E \rightarrow E_{1}$ называется $(k, \chi)$-ограниченным, если существует такая постоянная $k>0$, что для всех подмножеств $U$ из $E$ выполняется неравенство $\chi_{E_{1}}(A(U)) \leqslant k \chi_{E}(U)[3]$. Для любого ограниченного подмножества правильного пространства имеет место неравенство $\nu_{E}(U) \leqslant \chi_{E}(U)$, где $\nu_{E}(U)$ обозначает меру неравностепенной абсолютной непрерывности норм элементов подмножества $U$ правильного пространства $E: \nu_{E}(U)=\lim _{T \rightarrow \infty} \sup _{u \in U}\left\|P_{D\left(u, T, u_{0}\right)} u\right\|_{E}$ ([4]-[6]). Характеристика, подобная $\nu$ (для подмножеств пространства Лебега), впервые введена в [7] и независимо в [8]. Назовем подмножества $U_{1}$ и $U_{2}$ пространства $E$ сравнимыми, $U_{1} \leqslant U_{2}$, если в $E$ существует такая функция $b(s)$, что для любой функции $u \in U_{1}$ найдется функция $v \in U_{2}$, для которой п. в. выполняется неравенство $|u(s)| \leqslant|b(s)|+|v(s)|$. Аналогично, два оператора $F_{1}$ и $F_{2}$, действующих из правильного пространства $E$ в правильное пространство $E_{1}$, назовем сравнимыми, $F_{1} \leqslant F_{2}$, если $F_{1}(U) \leqslant F_{2}(U)$ для каждого подмножества $U$ из $E$. В силу того, что норма в правильном пространстве $E$ удовлетворяет условию $\|u\|_{E} \leqslant\|v\|_{E}$ для всех $v \in E$ и измеримых функций $u$, если $|u| \leqslant|v|$ п. в., и определения величины $\nu_{E}$

$$
U_{1} \leqslant U_{2} \Longrightarrow \nu_{E}\left(U_{1}\right) \leqslant \nu_{E}\left(U_{2}\right) \text {. }
$$

Уравнение вида $u=A(u ; \lambda)$, рассмотренное в теореме 1 настоящей работы, возникает во многих задачах нелинейной механики: отыскание критических нагрузок и форм потери устойчивости упругих систем, исследование автоколеба- 
тельных процессов, исследование процесса рождения волн и движущейся жидкости и т. Д. В подобных задачах роль параметра $\lambda$ могут играть нагрузки, частоты автоколебаний, скорости движения жидкости и т. п. [2]. В теореме 2 обобщен результат из [6], который, в свою очередь, обобщает еще более ранний результат автора настоящей работы об операторе Гаммерштейна (опубликован, в частности, в $[3,4.9 .1])$. Уравнение вида $u=K F(u)+f$, исследуемое в теореме 3, встречается в дифференциальной геометрии, физике, астрономии [9].

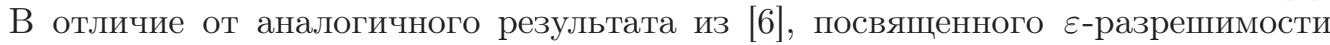
этого уравнения, здесь оператор $K$ не обязательно линейный.

\section{2. Формулировка результатов.}

Теорема 1. Пусть непрерывный оператор $A: E \rightarrow E$, где $E$ - правильное пространство, обладает свойством частичной аддитивности и $к$ тому же является вполне непрерывным как оператор из $M\left(u_{0}\right)$ в E. Пусть существует шар $B_{E}(f, r)$, для которого величина $\varkappa\left(B_{E}(f, r), A\right)$ конечна. Тогда существует такое число $\lambda_{0}>0$, что при всех $\lambda \leqslant \lambda_{0}$ уравнение $u=\lambda A u+f$ имеет хотя бъ одно решение.

Теорема 2. Пусть непрерывный оператор $K: L_{p}\left(\Omega, u_{0}\right) \rightarrow L_{q}\left(\Omega, u_{01}\right)(1 \leqslant$ $p<q<\infty)$ обладает свойством частичной аддитивности. Пусть, кроме того, $K$ является вполне непрерывным как оператор из $M\left(u_{0}\right)$ в $L_{q}\left(\Omega, u_{01}\right)$. Пусть непрерьвный оператор $F: L_{q}\left(\Omega, u_{01}\right) \rightarrow L_{p}\left(\Omega, u_{0}\right)$ для любой фучнкии $u \in L_{q}\left(\Omega, u_{01}\right)$ удовлетворяет условию

$$
|F(u)(s)| \leqslant|b(s)|+a u_{0}(s)\left|u(s) / u_{01}(s)\right|^{q / p},
$$

где $b(s) \in L_{p}\left(\Omega, u_{0}\right)$ - фиксированная функция и а - положительная постоянная. Тогда оператор $K F$ является $(k, \chi)$-ограниченным на каждом шаре $B_{L_{q}\left(\Omega, u_{01}\right)}\left(u_{1}, r\right)$ с постоянной $k=a \varkappa\left(F\left(B_{L_{q}\left(\Omega, u_{01}\right)}\left(u_{1}, r\right)\right), K\right) r^{q / p-1}$, если величина $\varkappa\left(F\left(B_{L_{q}\left(\Omega, u_{01}\right)}\left(u_{1}, r\right)\right), K\right)$ конечна. Кроме того, если существует производная Фреше оператора $K F$, то она вполне непрерьвна.

Теорема 3. Пусть выполнены предположения теоремы 2, причем для некоторого $r>0$ величина $\varkappa\left(F\left(B_{L_{q}\left(\Omega, u_{01}\right)}(\theta, r)\right), K\right)$ конечна. Пусть непрерывный оператор $F$ удовлетворяет условию $(2)$ с $b(s) \equiv \theta$.

1. Если $K(\theta)=\theta$, то существует такое $r_{0}>0$, что для любого $0<r \leqslant r_{0}$ u произвольной функиии $f \in L_{q}\left(\Omega, u_{01}\right)$ существует такое $\kappa>0$, что для произвольного измеримого подмножества $\widetilde{\Omega} \subseteq \Omega, \mu(\widetilde{\Omega})<\kappa$, уравнение $u=$ $P_{\widetilde{\Omega}} K F(u)+P_{\widetilde{\Omega}} f$ имеет хотя бы одно решение в шаре $B_{L_{q}\left(\Omega, u_{01}\right)}(\theta, r)$.

2. Если оператор $F$ частично аддитивен и $K(v)(s)=0$, когда $v(s)=0$, то для произвольной бункиии $f \in L_{q}\left(\Omega, u_{01}\right)$ уравнение $u=K F(u)+f$ имеет хотя бы одно решение $u \in L_{q}\left(\Omega, u_{01}\right)$.

3. Схемы доказательства результатов. В силу предположений относительно оператора $A$ и алгебраической полуаддитивности величин $\nu_{E_{1}}$ и $\chi_{E_{1}}$, учитывая определение величины $\nu_{E}(U)$ и формулу $(1)$, получаем следующее утверждение.

Лемма 1. Пусть оператор $A: E \rightarrow E_{1}$ переводит каждое множество, ограниченное в $\mathrm{M}\left(\mathrm{u}_{0}\right)$, в множество функиий с равностепенно абсолютно непрерывными нормами в $E_{1}$. Тогда для кажсдого ограниченного подмножества $U$ 
из $E$ имеет место неравенство $\nu_{E_{1}}(A(U)) \leqslant \varkappa(U, A) \nu_{E}(U)$, а если $A$ вполне непрерывен как оператор из $M\left(u_{0}\right)$ в $E_{1}$, mо $\chi_{E_{1}}(A(U)) \leqslant \varkappa(U, A) \nu_{E}(U)$.

Схема доказательства теоремы 1. Применяя лемму 1 и учитывая, что по предположению величина $\varkappa\left(B_{E}(f, r), A\right)$ конечна, доказываем, что оператор $\lambda A(u)+f$ является уплотняющим относительно $\chi$ на шаре $B_{E}(f, r)$. Утверждение теоремы 1 получаем из обобщения теоремы Шаудера на случай уплотняющих операторов [3, 1.5.11].

Оператор $F$, удовлетворяющий предположению $(2)$, сравним с оператором $F_{1}$, определенным формулой $F_{1}(u)(s)=a u_{0}(s)\left|u(s) / u_{01}(s)\right|^{q / p}, F \leqslant F_{1}$. Поэтому справедливо следующее утверждение:

Лемма 2. Пусть непрерывный оператор $F: L_{q}\left(\Omega, u_{01}\right) \rightarrow L_{p}\left(\Omega, u_{0}\right)$ удовлетворяет условию (2) для всех $u(s) \in L_{q}\left(\Omega, u_{01}\right)$, где a - положсительная постоянная, $b(s) \in L_{p}\left(\Omega, u_{0}\right)$ - фиксированная функиия. Тогда для любого подмножества $U \subset B_{L_{q}\left(\Omega, u_{01}\right)}\left(u_{1}, r\right)$ справедливо неравенство $\nu_{L_{p}\left(\Omega, u_{0}\right)}(F(U)) \leqslant$ $a r^{q / p-1} \nu_{L_{q}\left(\Omega, u_{01}\right)}(U)$.

Схемы доказательства теорем 2 и 3. В силу предположений относительно операторов и утверждений лемм 1 и 2 оператор $K F$ является $(k, \chi)$-ограниченным на каждом шаре $B_{L_{q}\left(\Omega, u_{01}\right)}\left(u_{1}, r\right)$ с $k=a \varkappa\left(F\left(B_{L_{q}\left(\Omega, u_{01}\right)}\left(u_{1}, r\right)\right), K\right) r^{q / p-1}$, если величина $\varkappa\left(F\left(B_{L_{q}\left(\Omega, u_{01}\right)}\left(u_{1}, r\right)\right), K\right)$ конечна. Завершается доказательство теоремы 2 применением рассуждений из [3, 1.5.9]. Первое утверждение теоремы 3 следует из обобщения теоремы Шаудера, так как в силу предположения относительно $F$, леммы 2 и теоремы $2 K F$ является $(k, \chi)$-ограниченным оператором с $k=a \varkappa\left(F\left(B_{L_{q}\left(\Omega, u_{01}\right)}(\theta, r)\right), K\right) r^{q / p-1}$ на шаре $B_{L_{q}\left(\Omega, u_{01}\right)}(\theta, r)$. Поэтому можно выбрать такое $r_{0}>0$, что для всех $0<r \leqslant r_{0}$ оператор $u \mapsto K F(u)+f$ является уплотняющим на шаре $B_{L_{q}\left(\Omega, u_{01}\right)}(\theta, r)$. Доказательство второго утверждения опирается на дополнительное предположение относительно $K$ и первое утверждение теоремы 3.

\section{ЛитеРАТУРА}

[1] П. П. Забрейко, Вестник Ярославского ун-та, 1974, № 8, $12-52$. [2] М. А. Красносельский, П. П. Забрейко, Е. И. Пустыльник, П. Е. Соболевский, Интегральные операторы в пространствах суммируемых функиий, Наука, М., 1966. [3] Р. Р. Ахмеров, М. И. Каменский, А. С. Потапов, А. Е. Родкина, Б. Н. Садовский, меръ некомпактности и уплотняюшие операторы, Наука, Новосибирск, 1986. [4] N. A. Yerzakova, Z. Anal. Anwendungen, 15:2 (1996), 299-307. [5] Н. А. Ерзакова, Сиб. матем. журн., 38:5 (1997), 1071-1073. [6] Н. А. Ерзакова, Научный вестник МГТУ ГА, сер. матем. физ., 2009, № 140, 57-64. [7] Н. А. Ерзакова, Об уплотняющих операторах в пространствах $L_{p}$, Деп. в ВИНИТИ 05.05.83, No. 2850-83, 1983. [8] J. Appell, E. De Pascale, Boll. Unione Mat. Ital. B, 3:2 (1984), 497-515. [9] N. J. Kalton, I. E. Verbitsky, Trans. Amer. Math. Soc., 351:9 (1999), 3441-3497.

Московский государственный технический университет гражданской авиации

Поступило в редакцию e-mail: naerz@mail.ru

31 июля 2009 г. 